\title{
Post-TAVI Follow-Up with MDCT of the Valve Prosthesis: Technical Application, Regular Findings and Typical Local Post-Interventional Complications
}

\section{Verlaufskontrolle nach TAVI mittels Multidetektor-CT der Klappenprothese: technische Durchführung, reguläre Befunde und typische lokale postinterventionelle Komplikationen.}

Authors

Martin Soschynski ${ }^{1}$, Fabio Capilli ${ }^{1}$, Philipp Ruile ${ }^{2}$, Franz-Josef Neumann ${ }^{2}$, Mathias Langer ${ }^{1}$, Tobias Krauss ${ }^{1}$

Affiliation

1 Department of Radiology, Medical Center - University of Freiburg, Faculty of Medicine, University of Freiburg, Germany, Freiburg, Germany

2 Department of Cardiology \& Angiology II, University Heart Center Freiburg-Bad Krozingen, Germany

Key words

TAVI, CT-spiral, post-interventional complications, leaflet thrombosis, annulus rupture

received 04.06 .2017

accepted 07.11.2017

Bibliography

DOI https://doi.org/10.1055/s-0043-124190

Published online: 28.12.2017

Fortschr Röntgenstr 2018; 190: 521-530

(c) Georg Thieme Verlag KG, Stuttgart · New York ISSN 1438-9029

Correspondence

Dr. Martin Soschynski

Radiology, University Hospital Freiburg, Hugstetter Straße 55, 79106 Freiburg, Germany

Tel.: ++49/7 61/27039540

Fax: ++49/7 61/27039500

martin.soschynski@uniklinik-freiburg.de

\section{ABSTRACT}

Background Transcatheter aortic valve implantation (TAVI) has evolved into an alternative procedure to surgical valve replacement for high-risk patients with aortic valve stenosis. Despite technical innovations, there is still a risk of complications during and after the intervention. After a TAVI procedure, ECG-gated multidetector computed tomography (MDCT) plays an important role in the early diagnosis of local complications. In this article, we explain for the first time how the technical acquisition of MDCT in the region of the aortic root is performed as post-interventional control of the TAVI prosthesis. In the second part normal post-interventional findings of different prosthetic valves as well as classic and uncommon complications in the implant area will be illustrated in several case studies.

Methods In this review the current literature from PubMed about ECG-gated MDCT after TAVI is summarized and structured. It is supplemented by several case studies from our institution.

Results and Conclusion Using retrospectively ECG-gated MDCT, an aortic valve prosthesis after TAVI can be visualized with high spatial resolution in several phases of the cardiac cycle. Images of the implanted aortic valve at all time points of the cardiac cycle enable a functional analysis of prosthetic leaflets similar to echocardiography. MDCT is superior to transthoracic echocardiography with respect to the direct detection of prosthetic leaflet thrombosis. The position of the device in relation to the coronary ostia and correct unfolding of the stent frame need to be evaluated. There are different types of stents carrying the valve leaflets with distinct ideal positions. Any stent should cover the left ventricular outflow tract (LVOT) along its whole circumference. Life-threatening complications in the implant area, such as annulus rupture, can be diagnosed reliably with CT.

Key points

- ECG-gated multidetector CT (MDCT) after transcatheter aortic valve implantation (TAVI) can provide early detection of postinterventional complications of the prosthetic valve and the aortic root.

- MDCT is superior to echocardiography with respect to the direct detection of prosthetic leaflet thrombosis.

- MDCT can also reveal hypokinesia of the thrombotic valve leaflets.

- Correct position of the device und unfolding of the stent frame differ according to the type of prosthesis.

- The integrity of the native aortic root should be carefully assessed. 


\section{Citation Format}

- Soschynski M, Capilli F, Ruile P et al. Post-TAVI Follow-Up with MDCT of the Valve Prosthesis: Technical Application, Regular Findings and Typical Local Post-Interventional Complications. Fortschr Röntgenstr 2018; 190: 521-530

\section{ZUSAMMENFASSUNG}

Hintergrund Die Transkatheter-Aortenklappen-Implantation (TAVI) hat sich als Alternative zum chirurgischen Klappenersatz für Patienten mit hohem Operationsrisiko etabliert. Trotz technischer Optimierungen besteht immer noch ein Risiko für Komplikationen während und nach dem Eingriff. Für die postinterventionelle Kontrolle der stentgetragenen Klappenprothese spielt die EKG-getriggerte Multidetektor-Computertomografie (MDCT) eine wichtige Rolle in der frühzeitigen Diagnostik von lokalen Komplikationen. In diesem Artikel gehen wir zunächst auf die technische Durchführung der MDCT im Bereich der Aortenwurzel zur Kontrolle der TAVIKlappenprothese ein. Im zweiten Schritt werden anhand mehrerer Fallbeispiele postinterventionelle Normalbefunde ver- schiedener Klappentypen sowie klassische und seltene Komplikationen im Implantationsgebiet illustriert.

Methode Die Übersichtsarbeit fasst die Literatur in PubMed zum Thema EKG-getriggerte MDCT nach TAVI strukturiert zusammen und wird um eine Fallsammlung aus unserem Institut ergänzt.

Ergebnisse und Schlussfolgerung Mittels retrospektiv EKGgetriggerter MDCT kann die Aortenklappenprothese nach TAVI räumlich hochaufgelöst in mehreren Phasen des Herzzyklus darstellt werden. Die Darstellung der Klappenprothese über den gesamten Herzzyklus erlaubt eine Funktionsanalyse der bewegten Klappe ähnlich der Echokardiografie. Die MDCT ist der transthorakalen Echokardiografie im direkten Nachweis einer Klappenthrombose überlegen. Bei der Befundung muss die Lage der stentgetragenen Klappe in Bezug zu den Koronargefäßen und die Stententfaltung evaluiert werden. Je nach Klappentyp bestehen unterschiedliche Stentformen und ideale Positionen. Jeder Stent sollte mit der gesamten Zirkumferenz dem linksventrikulären Ausflusstrakt anliegen. Lebensbedrohliche Komplikationen im Implantationsgebiet wie eine Annulusruptur können im CT sicher diagnostiziert werden.

\section{Introduction}

Transcatheter aortic valve implantation (TAVI) is an established alternative to surgical valve replacement for patients with a high surgical risk. Despite ever-growing experience, technical optimizations, and improved patient screening, there is still a risk of complications. ECG-triggered multidetector computed tomography (MDCT) is important for pre-interventional planning and can also be used for post-interventional early detection of both acute and long-term complications.

In the clinical routine, valve function is evaluated by means of transthoracic echocardiography. Paravalvular insufficiency can be detected with high sensitivity with the Doppler technique. Parameters such as the average pressure gradient and the velocity ratio across the valve prosthesis are used to detect stenosis. However, the valve leaflets often cannot be directly visualized on echocardiography [1, 2]. MDCT allows precise morphological visualization of the valve and analysis of movement throughout the entire cardiac cycle. Moreover, the stent position, coronary artery branches, and the integrity of the aortic root can be reliably evaluated. Reliable evaluation requires correct technical implementation of the CT scan, basic knowledge of the types of valves, normal post-interventional findings, and knowledge of classic and life-threatening complications after a TAVI procedure. This article discusses the fundamentals of the technical implementation and the evaluation of post-interventional MDCT.

\section{Implementation}

At our institute, MDCT of an aortic valve prosthesis is performed using the spiral technique under continuous table advance with retrospective ECG gating throughout the entire cardiac cycle with craniocaudal extension over the aortic root (Siemens Somatom Definition Flash, Siemens Healthcare, Forchheim, Germany) $[2,3]$. As a result, image reconstruction is possible for the relatively small scan volume with a craniocaudal length of approx. $8 \mathrm{~cm}$ in every phase of the cardiac cycle. If the heart valve is to be visualized with high resolution in every phase, the tube current modulation of the scanner must be switched off („pulsing off“). After bolus tracking in the left atrium $(100 \mathrm{HU})$ and a breathing command (7 seconds), the CT scan is initiated in the caudocranial direction. A bolus of $50 \mathrm{ml}$ of nonionic contrast agent (Imeron $400^{\circledR}$, Bracco) is injected at a rate of $4 \mathrm{ml} / \mathrm{s}$. This is followed by a $50-\mathrm{ml}$ saline bolus with an injection rate of $4 \mathrm{ml} / \mathrm{s}$.

In post-processing, the scan volume is reconstructed in increments of $50 \mathrm{~ms}$ or $5 \%$ of the cardiac cycle so that the scan volume is ultimately available for evaluation in up to 20 different phases with a slice thickness of $1 \mathrm{~mm}$.

\section{Radiological evaluation}

After data acquisition, the image data are sent to the picture archiving and communication system (PACS). With the help of dedicated image reporting software (we use SyngoVia workstation, Siemens Healthcare, Forchheim, Germany), the axial and sagittal viewing planes are aligned with respect to the stent axis so that the position of the valve prosthesis within the aortic root is optimally visualized ( $\triangleright$ Fig. $1 \mathbf{a}-\mathbf{c}$ ). As a result, the position of the stent frame, stent expansion, and the coronary artery branches can be evaluated and the examiner can assess the valve leaflets, general complications, such as hematomas, and the intactness of the aortic annulus. To detect thickening of the valve leaflets and to check leaflet movement, all phases of the cardiac cycle should be evaluated. The multiphase view in image reporting software in 


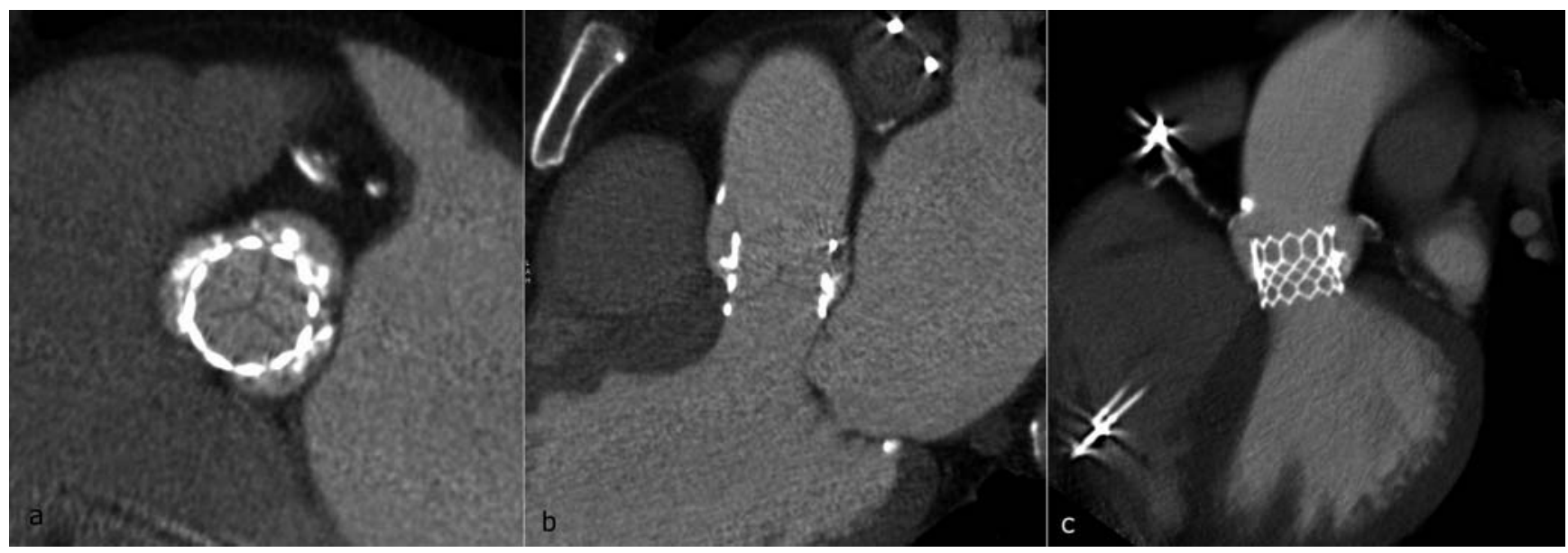

- Fig. 1 Typical post-interventional MDCT of an Edwards SAPIEN 3-prosthesis in a axial and b, c parasagittal angulation to the prosthetic valve. a In the axial view the native valve leaflets can be seen as fine linear hypodensities at the level of the aortic bulbus. At this level the prosthesis is usually not fully attached to the aortic wall. b Parasagittal reconstruction shows the correct craniocaudal position of the prosthesis and complete attachment of the stent to the aortic wall at the level of the aortic annulus. cThick-slab multiplanar reconstruction (MPR) illustrates the stent geometry and the exact implantation position.

which the individual phases can be viewed in the desired angulation as a video is suitable for this purpose. Valve leaflet movement can thus be evaluated dynamically $(4 \mathrm{D}) \triangleright$ Video 1.

\section{Normal findings}

\section{Stent frame}

The valve types most commonly implanted at our hospital are the „Edwards SAPIEN XT“ and its next generation the „Edwards SAPIEN 3“ from Edwards Lifesciences (Irvine, California, USA), the "CoreValve“ and its next generation the "CoreValve Evolut R“ from Medtronic (Minneapolis, Minnesota, USA) and the „Lotus“ from Boston Scientific (Natick, Massachusetts) [3].

\section{Edwards SAPIEN prostheses}

The „Edwards SAPIEN XT“ and the „Edwards SAPIEN 3“ are balloon-expandable aortic valve prostheses consisting of a cobaltchromium stent with bovine pericardial leaflets. The lower 2/3 of the stent has a sealing cuff. The upper portion of the stent frame is open [4].

The typical post-interventional CT finding for an Edwards SAPIEN 3 prosthesis is shown in - Fig. $\mathbf{1 a - c . ~ I n ~ t h e ~ c a s e ~ o f ~ b a l - ~}$ loon-expandable aortic valve prostheses like the Edwards SAPIEN prosthesis, the stent frame is designed to expand fully in a circular fashion so that it presses the calcified original valve against the wall of the aortic root [4].

The best clinical successes are achieved when the stent frame is fixed at the level of as well as under the insertion of the original semilunar leaflets. Therefore, the original recommendation is to place the prosthesis so that the middle of the stent is at the level of the original annulus so that $50 \%$ of the stent frame extends proximally and $50 \%$ distally [5]. In the case of Edwards SAPIEN prostheses, upward movement of the stent can occur during

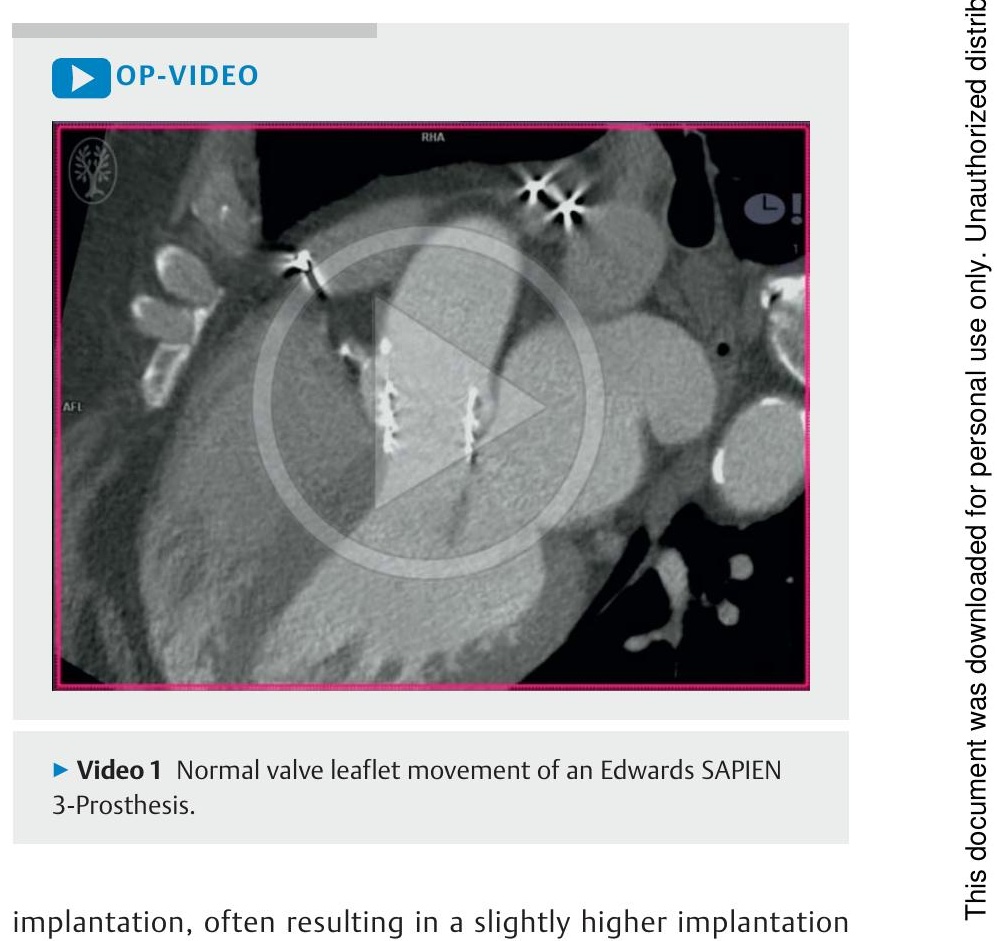

implantation, often resulting in a slightly higher implantation position of the valve [6]. This higher implantation position is still associated with a good clinical result. A good result is therefore achieved when the inferior stent end is implanted below the annulus, i. e. below the semilunar leaflet insertion, and the cranial stent end completely covers the semilunar leaflets [4]. In the case of a position that is too high, the basal insertion of the original valve cannot be covered while in the case of a position that is too low, the free edge of the original valve (lunula) cannot be completely covered. The stent seal cannot be seen on CT. The covered part cannot be differentiated from the uncovered part.

Even in the case of optimal positioning, the stent frame can extend partially over the coronary ostia, usually the left. In the case of a valve position that is too high, the stent frame fully cov- 

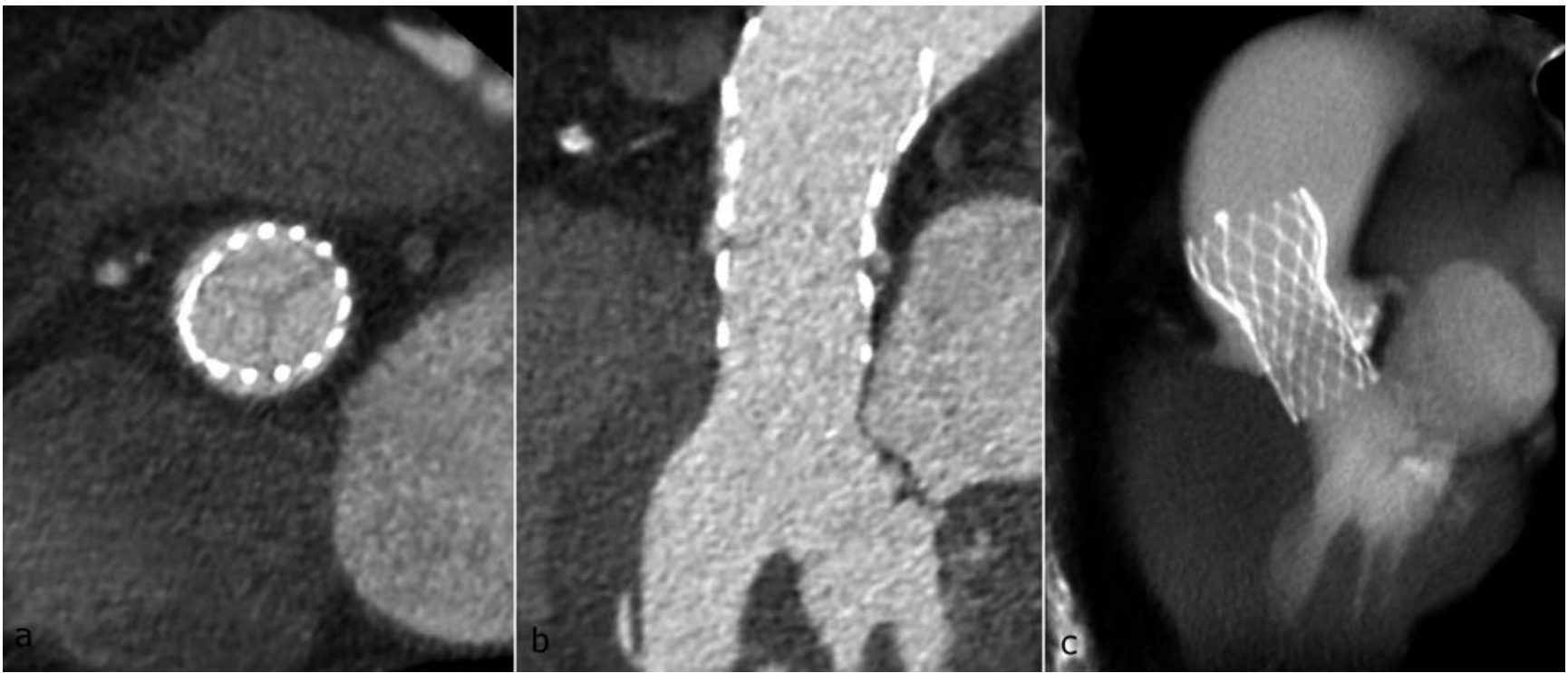

- Fig. 2 Normal CT finding of a CoreValve Evolut R-prosthesis in a axial and $\mathbf{b}$ parasagittal angulation as well as $\mathbf{c}$ thick-slab multiplanar reconstruction (MPR) illustrating the stent geometry and the exact implantation position.

ers a coronary ostium, usually the left. However, since only the inferior 2/3 of the stent is covered with a seal, occlusion of the coronary ostia almost never occurs [4]. Normal contrast enhancement of the free coronary ostia should be present.

The entire circumference of the stent should lie against the left ventricular outflow tract (LVOT) ( $\triangleright$ Fig. 1b, c). Massive, asymmetrical calcifications can result in incomplete conformation of the stent to the wall of the LVOT [4].

\section{CoreValve prostheses}

The CoreValve prostheses from Medtronic consist of a cup-shaped nitinol stent with porcine pericardial leaflets. In contrast to Edwards SAPIEN prostheses, these prostheses expand automatically after release. Therefore, they do not need to have a round shape after implantation but rather conform to the shape of the annulus [4]. They are significantly longer than Edwards SAPIEN prostheses and have a broader distal anchoring point in the ascending aorta but do not form a seal there ( $\triangleright$ Fig. 2a-c) [7]. The optimal position is with the lower end approximately $4-6 \mathrm{~mm}$ below the original annulus level [5]. The position is too low when the lower end is more than $12 \mathrm{~mm}$ below the annulus and the position is too high when the lower end is above the annulus [8].

\section{Lotus prosthesis}

The Lotus prosthesis from Boston Scientific also consists of a nitinol frame with a round shape when expanded and valve leaflets of bovine pericardium. A flexible polyurethane/polycarbonate seal around the lower half of the stent frame (adaptive seal) prevents paravalvular leaks [9]. In contrast to the other two prostheses, the Lotus valve prosthesis is expanded mechanically via three arms and not automatically or via balloon catheter. This allows relatively controlled insertion of the valve and also the option of retrieving and repositioning the valve even after complete expansion. An additional radiopaque tantalum marker in the center of the stent frame facilitates positioning of the valve [9]. During expansion of the stent, the stent frame shortens and the marker moves in the direction of the annulus until it reaches the correct position at the level of the sinus of Valsalva, approx. $5 \mathrm{~mm}$ distal to the annulus [10]. The marker can cause beam-hardening artifacts on CT and must not be confused with a para- or intravalvular foreign object ( $\triangleright$ Fig.3a-c).

\section{Prosthesis leaflets}

The prosthesis leaflets of the newly implanted aortic valve are visible on CT as fine, symmetrical, hypodense, arched structures within the lumen ( $\vee$ Fig. 1a, b). Thickening of one or more leaflets is to be categorized as pathological.

The multi-phase reconstruction from the ECG-triggered CT dataset allows evaluation not only of the morphology of the prosthesis leaflets but also of valve movement. In particular, in areas with a thickened valve leaflet, hypokinesia, i. e., reduced movement between diastole and systole, must be evaluated. Dedicated image reporting software can be used to visualize leaflet movement as a video sequence $>$ Video 1 .

\section{Aortic root}

Both the valve prosthesis and the integrity of the aortic root must be assessed. The shape of the aortic root can change post-interventionally from elliptical to round. However, an eccentric aneurysm (pseudoaneurysm, refer to Chapter V, section 6 „Annulus rupture" with figures) or fluid around the aortic root must not be present [11].

\section{Special case: transapical TAVI}

After transapical TAVI with access via the cardiac apex, hyperdense suture material (felt) is typically located around the exterior of the cardiac apex ( $\downarrow$ Fig. 4a). This must not be confused with 

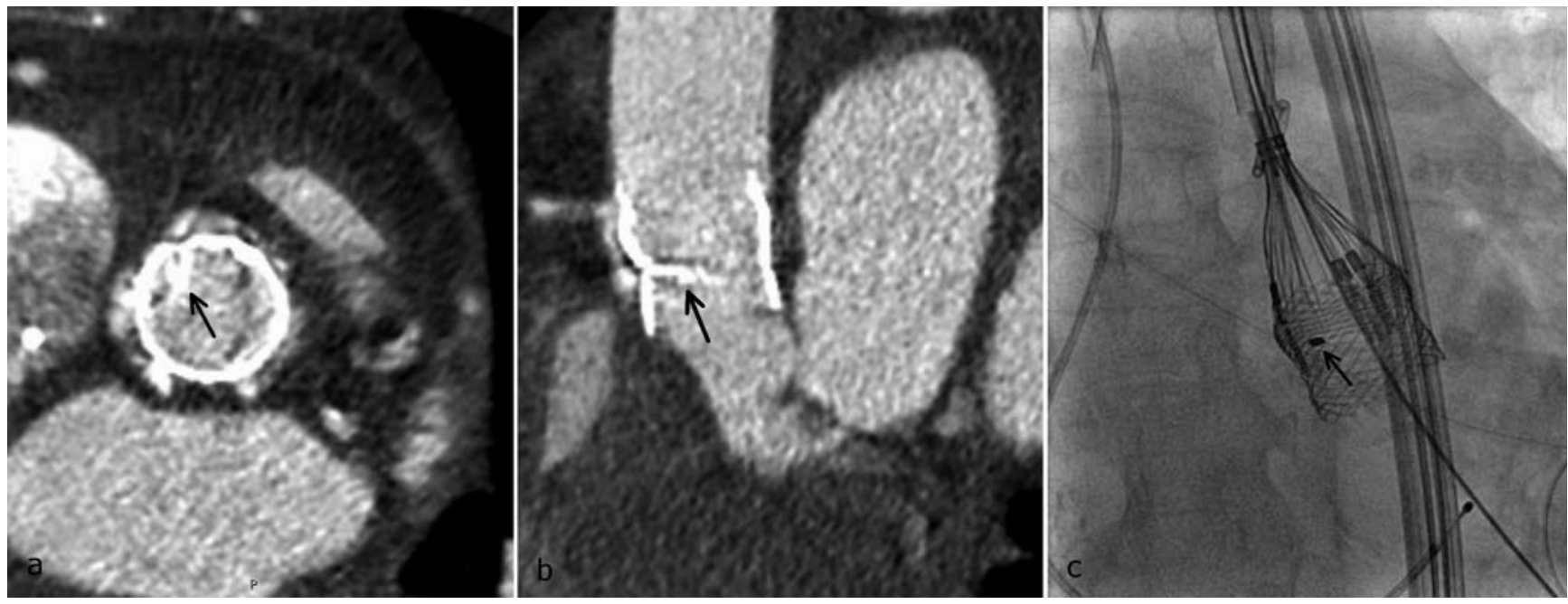

- Fig. 3 Normal CT findings of a Lotus-prosthesis in a axial and $\mathbf{b}$ parasagittal angulation as well as $\mathbf{c}$ fluoroscopy during implantation. Note the radiopaque marker which causes beam hardening artifacts on CT (arrows). After deployment, the correct position of the marker is at the level of the sinus of Valsalva, about $5 \mathrm{~mm}$ distal to the native annulus.

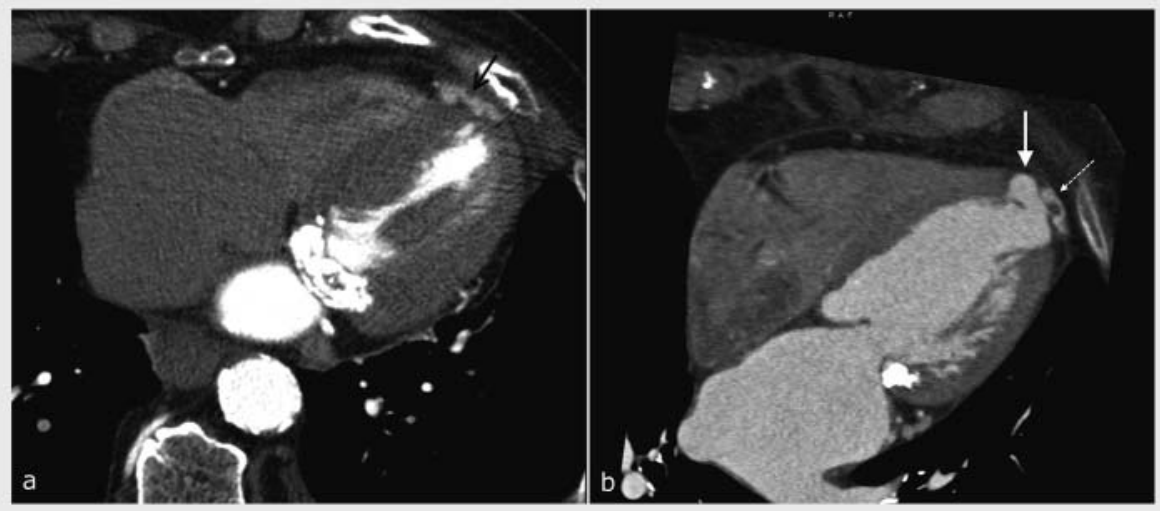

- Fig. 4 a Felt around the cardiac apex (arrow) after transapical TAVI, not to be confused with a suture aneurysm. $\mathbf{b}$ Suture aneurysm at the cardiac apex after transapical TAVI (thick arrow). Laterally hyperdense felt material (dashed arrow).

the rare complication of a suture aneurysm after transapical TAVI ( Fig. 4b) [4, 12]. Purely on the basis of density, felt material may not be able to be differentiated from a pseudoaneurysm in the arterial phase. However, the typical course and configuration of the felt material around the cardiac apex usually allows differentiation. In the case of uncertainty or primary suspicion of a suture aneurysm, a native ECG-triggered low-dose CT scan (as in calcium scoring in coronary imaging) can be additionally performed here. After transapical TAVI, mediastinal air inclusions and minimal mediastinal fluid are common postoperative findings and have no pathological significance.

\section{Complications}

\section{Valve thrombosis}

The complication most commonly observed on post-interventional CT is valve thrombosis. The incidence of early valve thrombosis is significantly higher than previously assumed, i. e., 5-10\% in post-TAVI follow-up studies using MDCT [1, 3]. The following must be taken into consideration in the evaluation: The leaflets of all types of prostheses are very thin, typically thinner than surgical valves $(<0.35 \mathrm{~mm})$. They may only be visible on CT as fine hypodense lines. Even the most minor thickening can no longer be classified as „normal“. The leaflets are therefore not measured on CT. A cut-off value is not described in the literature and thickening is described on a purely visual basis. For differentiation from an artifact, hypodense thickening of the leaflet is defined as true valve thickening when it can be visualized in multiplanar reconstructions (MPRs) on at least 2 planes and in at least 2 phases of 


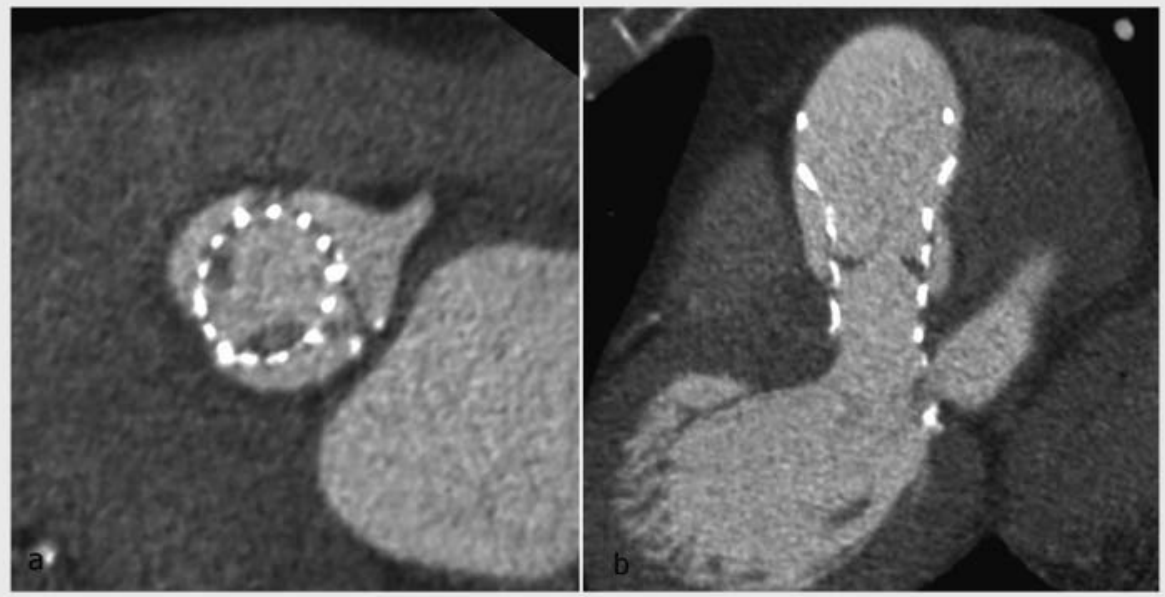

Fig. 5 Thickening of the attachments of the right and non-coronary leaflet in a axial and $\mathbf{b}$ parasagittal view, which is typical for leaflet thrombosis.

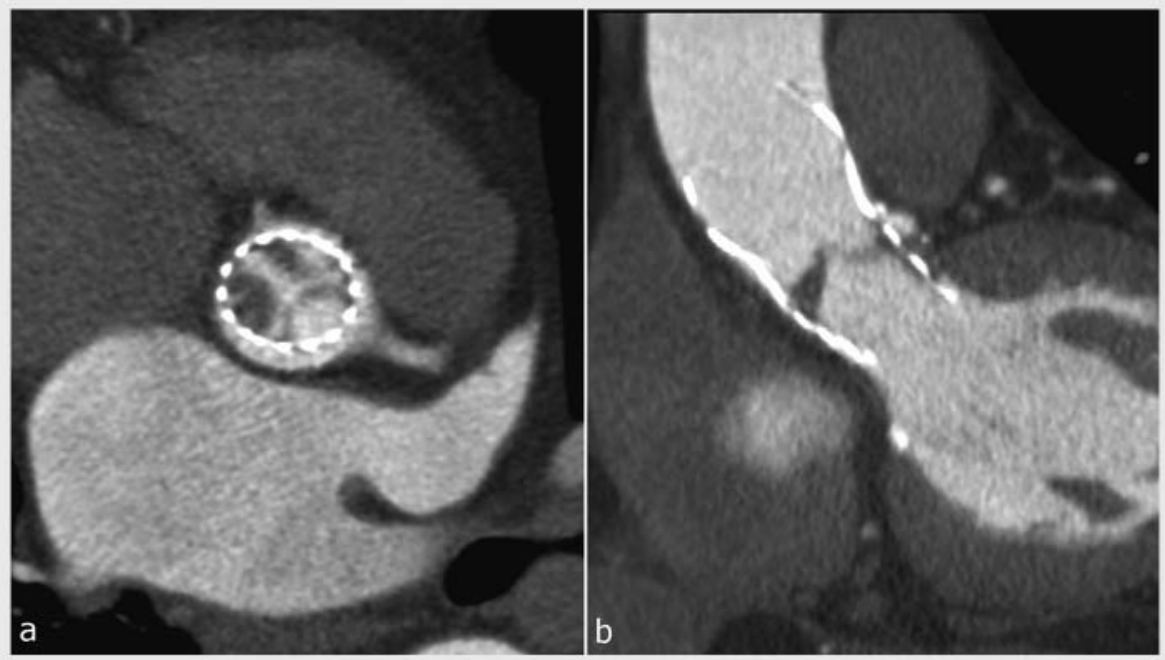

- Fig. 6 Severe leaflet thrombosis of a CoreValve-prosthesis with profuse thickening mainly of the right and non-coronary leaflet a axial and b parasagittal. Concomitant marked hypokinesia which echocardiographically manifested as valve stenosis.

the cardiac cycle ( $\triangleright$ Fig. $5 \mathbf{a}, \mathbf{b}, \mathbf{6 a}, \mathbf{b})[2,3]$. Thrombosis of the valve leaflet can be but is not necessarily associated with hypokinesia of the corresponding valve leaflet. One of more leaflets can be affected $\nabla$ Video 2 .

With respect to differential diagnosis, thickening of a leaflet, particularly at the base, tends to indicate valve thrombosis while thickening of prosthesis leaflets with free-floating structures indicates the rare complication of infectious endocarditis $[3,13,14]$.

Early, small thromboses are often asymptomatic. In transthoracic echocardiography, an increased average pressure gradient and an increased velocity ratio over the valve prosthesis indicate stenosis, often caused by thrombosis [15]. However, minor valve thromboses are not necessarily associated with hypokinesia and thus also not with stenosis. Therefore, they are usually not visible on transthoracic echocardiography [1]. Even with transesophageal echocardiography, thrombosis cannot be visualized due to through-transmission artifacts caused by the TAVI stent [1, 2].

The treatment of clinically occult valve thromboses after TAVI with anticoagulants is controversial [3].

\section{Incorrect valve position}

As explained in the section "Normal findings", the prosthesis should ideally be positioned so that the covered portion of the stent fully covers the original valve. If this is not the case, the original valve leaflets project into the lumen, resulting in an obstruction ( $\triangleright$ Fig. 7). Moreover, the incorrect stent position can present an increased risk for secondary dislocation ( $\triangleright$ Fig. 8). 


\section{OP-VIDEO}

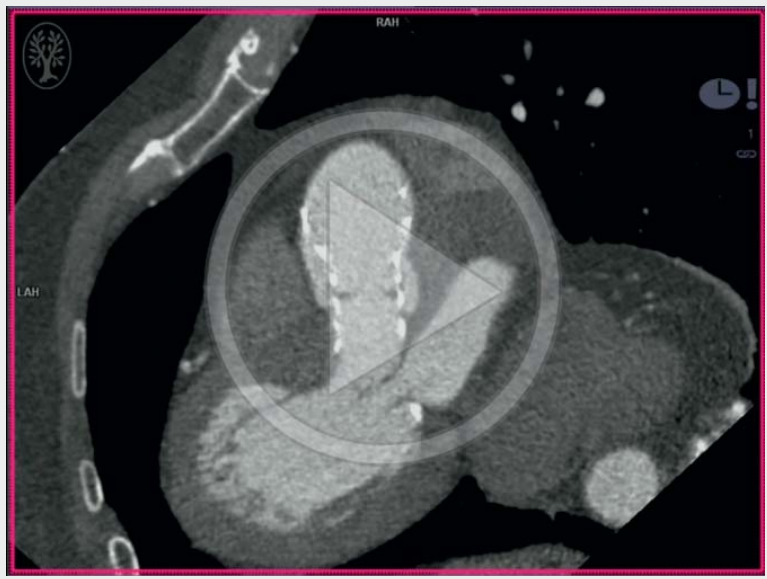

- Video 2 Same patient as in > Fig. 4. Thrombotic apposition of right and non-coronary leaflet. There is hypokinesia at the thickened areas.

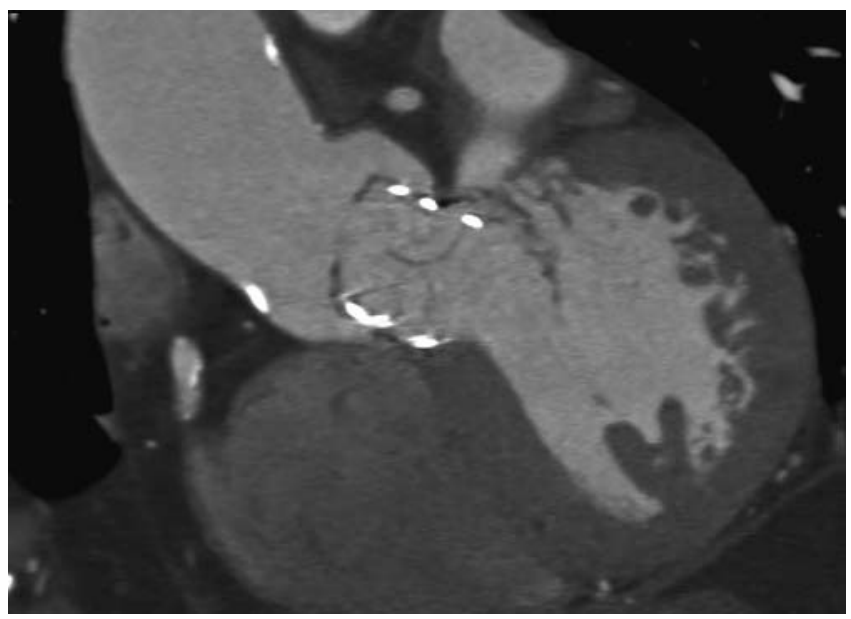

- Fig. 7 Position of an Edwards SAPIEN 3-prosthesis in the LVOT that is too low with the original valve leaflets extending beyond the stent frame, fortunately not causing significant obstruction.

\section{Stent dislocation}

Stent dislocation after correct initial positioning is a rare complication and usually occurs during the intervention $(0.2 \%$ of patients [16]). If the position of the valve prosthesis is too low with hemodynamically relevant paraaortic regurgitation, an attempt is made during the intervention to reposition the valve manually with a special catheter technique (lasso technique) [8]. If that attempt is unsuccessful, a second valve can be inserted into the first in a slightly more distal position to prevent paravalvular regurgitation.

In the case of a valve prosthesis that is initially implanted too high or in the case of secondary dislocation in a distal direction with hemodynamic relevance or overlap of the coronary ostia, an attempt is made to reposition the valve further distally with

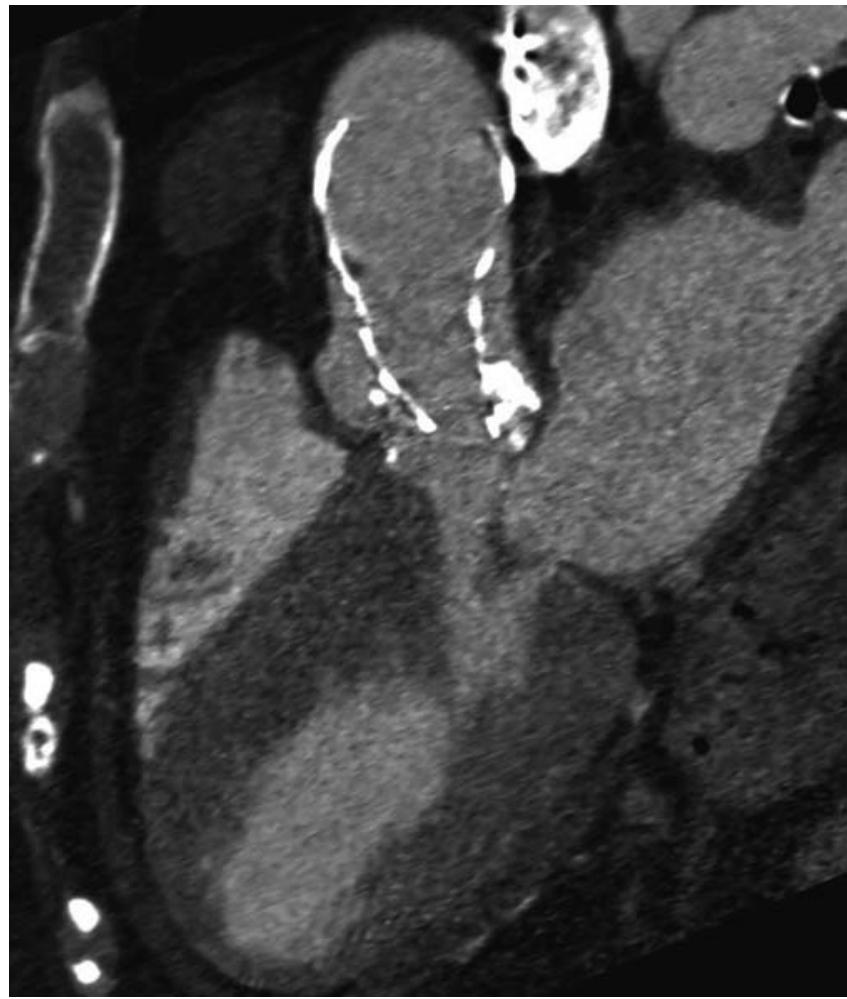

- Fig. 8 Position of a CoreValve-prosthesis that is too high in the aortic root. Due to pronounced confluent calcifications of the original valve, the prosthesis could not be placed deeply enough. Consequently, the caudal end of the stent frame only barely extends to the annulus. The stent wire is not fully unfolded. The risk of secondary dislocation into the ascending aorta is increased.

respect to the annulus into the ascending aorta to create space for a second valve prosthesis and to correctly position it [8].

\section{Occlusion of the coronary ostia}

Occlusion of the coronary ostia due to a particularly high stent position is very rare since only the lower stent portions are covered in the Edwards SAPIEN and Lotus prostheses and there is significant tapering at the level of the coronary ostia in the case of the CoreValve prosthesis $[4,9,12]$. Occlusion of the ostia can be caused not only by the stent but also by positioning of the original calcified valves in front of the ostium, an aortic dissection with involvement of the coronary artery, or embolization of calcium or deposits on the old aortic valve into the coronary arteries. However, it is a very rare acute complication that is typically diagnosed during the intervention via angiography or echocardiography and is only seen in extreme exceptions on CT [17 - 19]. The total incidence in studies is $0.66 \%$ [20]. Acute occlusion could be treated by catheter in most cases, with open bypass operation being necessary in only a few cases $[19,20]$. For protection, in high-risk cases, catheterization with a coronary catheter is recommended prior to insertion of the valve. The use of a retractable valve (e.g. Lotus) can also be helpful [20]. 


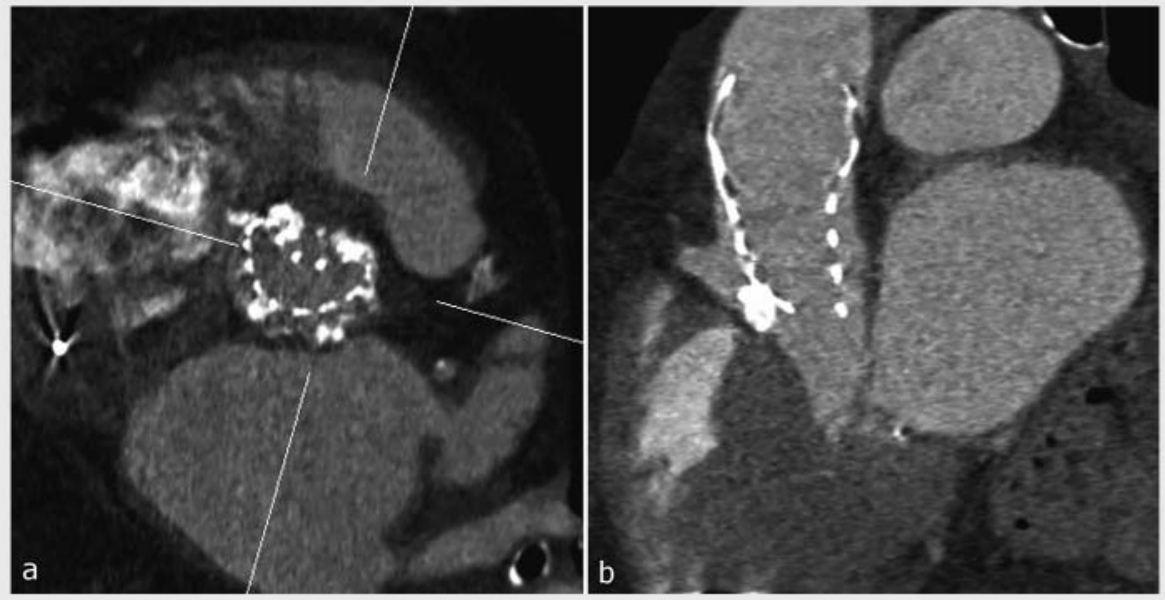

- Fig. 9 Same patient as in Fig. 8 Pronounced confluent calcifications of the original valve cause impression of the stent frame a which is not fully adapted to the annuls $\mathbf{b}$. This results in paravalvular insufficiency.
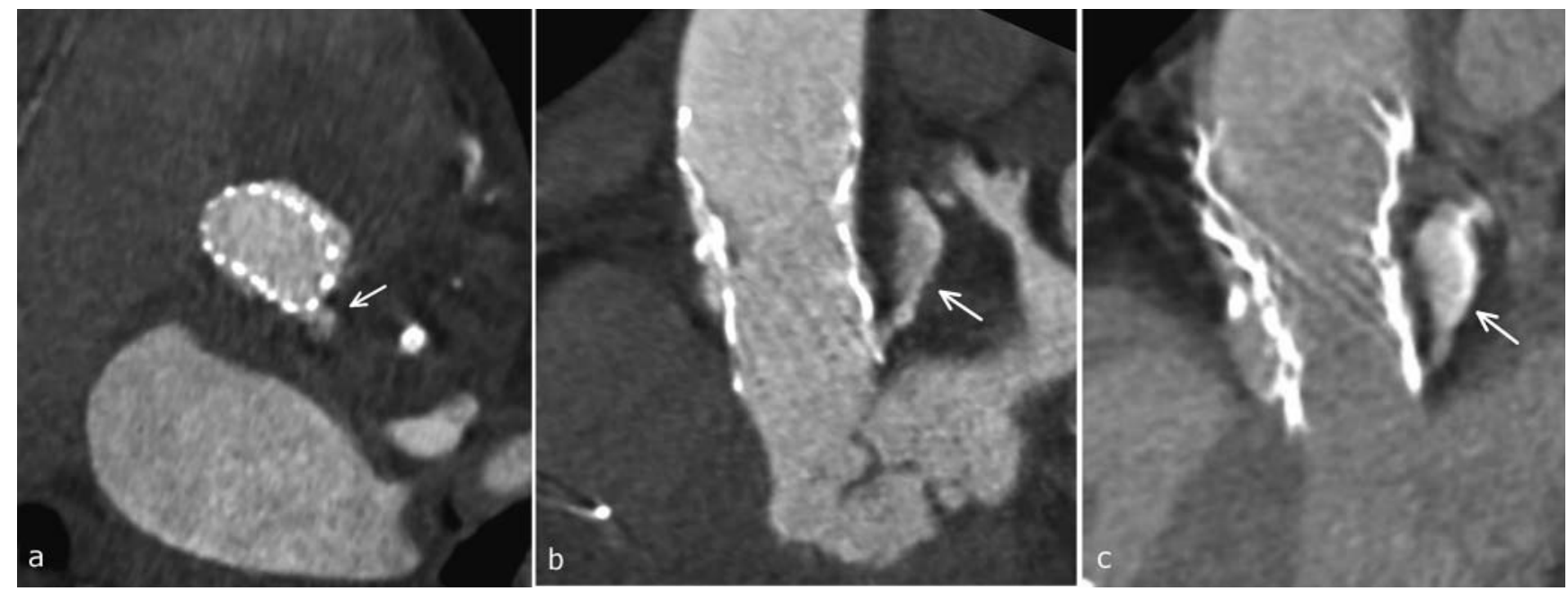

- Fig.10 Acute rupture of the annulus (arrows). a Axial slice at the level of the annulus showing the origin of contrast extravasation from the aortic root under the left coronary cusp. $\mathbf{b}, \mathbf{c}$ Parasagittal view of the whole contrast depot between the aortic root and the left atrium in $\mathbf{b}$ early arterial and $\mathbf{c}$ venous contrast phase, consistent with contained rupture.

\section{Incorrect stent expansion}

Pronounced, asymmetrical calcification of the original aortic valve rarely results in incomplete stent expansion. It usually leads to incomplete conforming of the stent to the LVOT, resulting in paravalvular regurgitation ( $\triangleright$ Fig.9a, b) [4]. However, optimal positioning and expansion of the valve prosthesis does not rule out paravalvular insufficiency. In contrast, in our experience, most cases of paravalvular insufficiency seen on echocardiography cannot be visualized on MDCT even retrospectively. The Doppler echocardiography technique provides significantly more sensitive detection of paravalvular insufficiencies than MDCT.

\section{Annulus rupture - rare but life-threatening complication}

A life-threatening post-interventional complication is rupture of the aortic annulus. One risk factor is significant calcification of the left-ventricular outflow tract [21]. MDCT shows contrast agent extravasation. This can be in the form of contrast pooling or a pseudoaneurysm in terms of a covered rupture ( $\triangleright$ Fig. 10ac, $11 \mathbf{a}-\mathbf{c}$ ). A mediastinal hematoma is typically also present. In the case of a perforation in the pericardium, a hemopericardium can result in pericardial tamponade.

\section{Discussion - indication for post-TAVI MDCT}

There is currently no recommendation for the routine use of MDCT in all patients after TAVI. This is a subject of controversy in the literature. To date, studies have shown that small thromboses 

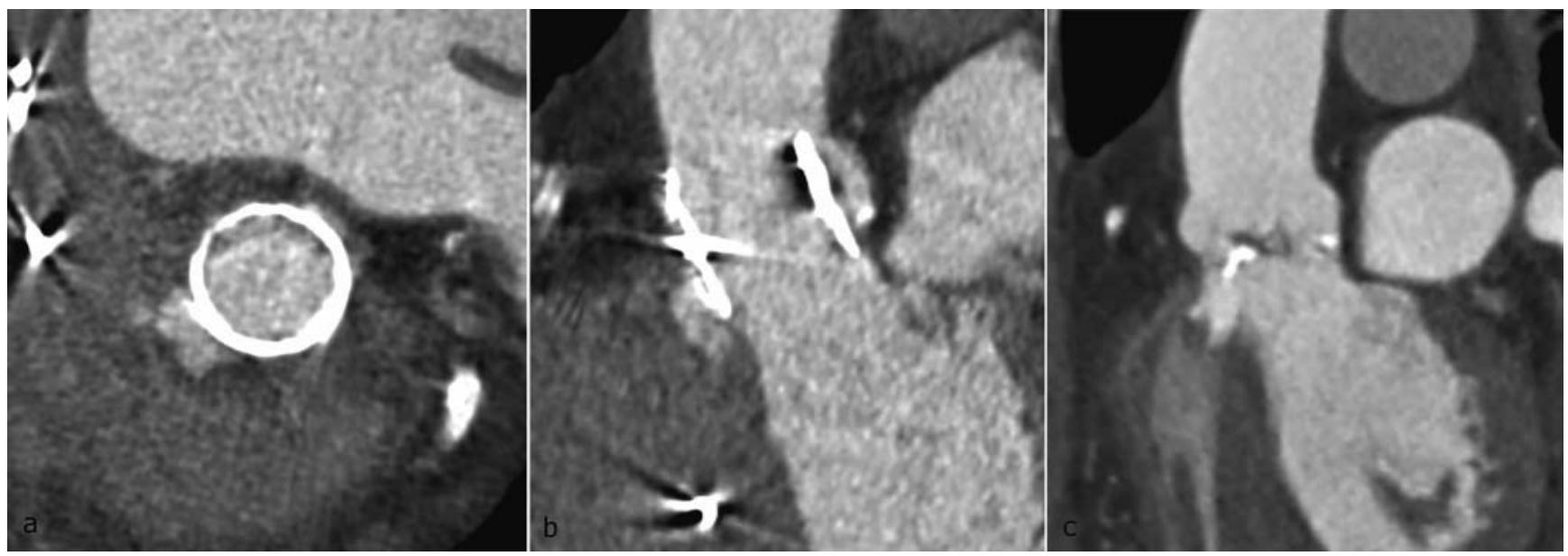

- Fig. 11 This figure does not show post-interventional annulus rupture. The eccentric pseudoaneurysm shown in a, b cannot be differentiated from a contained annulus rupture on the basis of the imaging alone. It existed before the intervention (already apparent on the pre-TAVI CT c). It corresponds to an old paravalvular abscess after endocarditis which is now perfused as a pseudoaneurysm. In spite of the pseudoaneurysm, TAVI could be performed without any complications. The patient was free of symptoms at the time of post-TAVI CT.

detected by MDCT are usually clinically unapparent. They are not necessarily associated with functional impairment of the valve or cardiac insufficiency [3, 22]. The use of anticoagulation therapy in these cases is questioned in the literature [3]. In the entire TAVI patient population, late dislocation of the stent $(0.2 \%$ of patients [16]) and coronary artery occlusion ( $0.66 \%$ of patients [20]) are extremely rare complications that probably would not justify routine use of CT after TAVI in asymptomatic patients. Paravalvular insufficiencies are also not a primary indication for computed tomography since they can usually be effectively diagnosed by echocardiography as described above.

However, one study including 135 patients who all underwent a routine follow-up MDCT examination after TAVI showed major complications in $4-5 \%$ of the patients. These included rupture of the sinus of Valsalva, aortic dissection, intramural hematoma, and left-ventricular pseudoaneurysm [23]. These complications were asymptomatic in 4 patients and were first detected on MDCT. Moreover, studies showed that early valve thrombosis in the first three months after TAVI is more common than previously assumed (4-10\% of patients) [1, 3]. Without anticoagulation therapy or with only dual antiplatelet therapy, thrombosis typically progresses and can lead over time to significant movement restrictions resulting in hemodynamically relevant valve stenosis. Anticoagulation therapy with phenprocoumon plus clopidogrel resolved the valve thrombosis. Therefore, patients with minor, subclinical valve thromboses do not require anticoagulation therapy but should be closely monitored [3].

The use of post-TAVI MDCT for further clarification in the case of new or progressing functional impairment of the valve, clinical worsening, or unclear echocardiographic and clinical findings is largely accepted.

Of course, the pretest probability of complications is significantly higher for these patients after examination by cardiologists and interdisciplinary consultation than for the total patient population (in percent, see above).
In rare cases in which a serious complication, such as annulus rupture, is suspected during the intervention, a post-TAVI MDCT scan should always be performed as quickly as possible.

Post-TAVI MDCT is a quick and relatively safe method for this purpose and uses a comparatively small amount of contrast agent. Although the dose is relatively high (in studies with retrospective gating approx. $16 \mathrm{mSv}$ with a scan length of $15 \mathrm{~cm}$ ) [24], it should be taken into consideration that patients in whom a TAVI prosthesis was implanted tend to be of an advanced age and usually have comorbidities. However, retrospective gating with data acquisition over the entire cardiac cycle is not necessary for all issues. Prospective ECG gating with data acquisition can only be performed within a narrow time frame, e. g., during diastole, to rule out aortic dissection or an incorrect stent position. As a result the dose can be significantly reduced. However, the disadvantage of prospective gating is that the option to reconstruct the raw data at different points in time in the ECG cycle (selection of the „best“ reconstruction phase) or to change ECG synchronization points as desired is not available to the same extent as in the case of retrospective gating. In the case of arrhythmia, these options in retrospective gating can significantly improve image quality.

\section{Conclusion}

Using only a small amount of contrast agent, an aortic valve prosthesis can be effectively visualized with high spatial resolution in multiple phases of the cardiac cycle via retrospective ECG-triggered MDCT after transcatheter aortic valve implantation (TAVI). Requirements for the reliable evaluation of MDCT are basic knowledge of the types of valves and knowledge of normal post-interventional findings as discussed in this article. MDCT is superior to echocardiography in the direct detection of valve thrombosis. By recording multiple phases of the cardiac cycle, a functional analysis of the valve and thus detection of hypokinesia in the case of 
valve thrombosis are possible. The position of the stent-bearing valve, stent expansion, and the integrity of the aortic root must be evaluated after the intervention. Life-threatening complications, such as annulus rupture, can be reliably diagnosed on CT.

\section{Conflict of Interest}

The authors declare that they have no conflict of interest.

\section{Literatur}

[1] Leetmaa T, Hansson NC, Leipsic ] et al. Early Aortic Transcatheter Heart Valve Thrombosis: Diagnostic Value of Contrast-Enhanced Multidetector Computed Tomography. Circ Cardiovasc Interv 2015; 8: e001596. doi:10.1161/CIRCINTERVENTIONS.114.001596

[2] Pache G, Schoechlin S, Blanke P et al. Early hypo-attenuated leaflet thickening in balloon-expandable transcatheter aortic heart valves. European Heart Journal 2016; 28: 2263-2271

[3] Ruile P, Jander N, Blanke P et al. Course of early subclinical leaflet thrombosis after transcatheter aortic valve implantation with or without oral anticoagulation. Clinical Research in Cardiology 2016; 106: 85-95

[4] Blanke P, Schoepf U, Leipsic ] et al. CT in Transcatheter Aortic Valve Replacement. Radiology 2013; 3: 650-669

[5] Litmanovich DE, Ghersin E, Burke DA et al. Imaging in Transcatheter Aortic Valve Replacement (TAVR): role of the radiologist. Insights into Imaging 2014; 1: 123-145

[6] Dvir D, Lavi Ifat et al. Multicenter Evaluation of Edwards SAPIEN Positioning During Transcatheter Aortic Valve Implantation With Correlates for Device Movement During Final Deployment. JACC: Cardiovascular Interventions 2012; 5: 563-570

[7] Jilaihawi H, Chin D, Spyt T et al. Prosthesis-patient mismatch after transcatheter aortic valve implantation with the Medtronic-Corevalve bioprosthesis. European Heart Journal 2010; 7: 857-864

[8] Laborde JC, Breaker S], Roy D et al. Complications at the time of transcatheter aortic valve implantation. Methodist Debakey Cardiovasc J 2012; 8: 38-41

[9] Halapas A, Chrissoheris M, Bouboulis N et al. Update on current TAVI technology, indications, screening, and outcomes. Continuing Cardiology Education 2016; 1: 37-46

[10] De Backer O et al. Efficacy and safety of the Lotus Valve System for treatment of patients with severe aortic valve stenosis and intermediate surgical risk: Results from the Nordic Lotus-TAVR registry. International Journal of Cardiology 2016; 219: 92 - 97
[11] Leipsic ], Gurvitch R, LaBounty TM et al. Multidetector Computed Tomography in Transcatheter Aortic Valve Implantation. JACC: Cardiovascular Imaging 2011; 4: 416-429

[12] Vanezis AP, Baig MK, Mitchel IM et al. Pseudoaneurysm of the left ventricle following apical approach TAVI. Journal of Cardiovascular Magnetic Resonance 2011; 1: 79

[13] Lane AB, Cahill MS, Letizia AG et al. Multimodality imaging of multivalvular endocarditis after transcatheter aortic valve replacement. Journal of Cardiovascular Computed Tomography 2015; 1: 68-70

[14] Entrikin DW, Gupta P, Kon ND et al. Imaging of infective endocarditis with cardiac CT angiography. Journal of Cardiovascular Computed Tomography 2012; 6: 399-405

[15] Spethmann S, Dreger H, Schattke $S$ et al. Doppler haemodynamics and effective orifice areas of Edwards SAPIEN and CoreValve transcatheter aortic valves. European Heart Journal - Cardiovascular Imaging 2012; 8: $690-696$

[16] Uzoma N et al. Review of Reported Causes of Device Embolization Following Trans-Catheter Aortic Valve Implantation. Am J Cardiol 2015; 115: $1767-1772$

[17] Kuck KH, Eggebrecht H, Figulla HR et al. Qualitätskriterien zur Durchführung der transvaskulären Aortenklappenimplantation (TAVI). Der Kardiologe 2015; 1: 11-26

[18] Ribeiro HB, Webb JG, Makkar RR et al. Predictive Factors, Management, and Clinical Outcomes of Coronary Obstruction Following Transcatheter Aortic Valve Implantation. Journal of the American College of Cardiology 2013; 17: $1552-1562$

[19] Ribeiro HB, Sarmento-Leite R et al. Coronary Obstruction Following Transcatheter Aortic Valve Implantation. JACC Cardiovascular Interventions 2013; 6: 452-461

[20] Takahide A et al. Incidence and Predictors of Coronary Obstruction Following Transcatheter Aortic Valve Implantation in the Real World. Catheterization and Cardiovascular Interventions 2017; 90: 1192-1197

[21] Hayashida K, Bouvier E et al. Potential Mechanism of Annulus Rupture During Transcatheter Aortic Valve Implantation. Catheterization and Cardiovascular Interventions 2013; 82: E742-E746

[22] Jeroen J et al. Does computed tomography detect bioprosthetic aortic valve thrombosis? New findings, new questions? European Heart Journal 2016; 37: 2272-2275

[23] Yuki K et al. Impact of Subclinical Vascular Complications Detected by Systematic Postprocedural Multidetector Computed Tomography After Transcatheter Aortic Valve Implantation Using Balloon-Expandable Edwards SAPIEN XT Heart Valve. Am J Cardiol 2017; 119: 1100-1105

[24] Kristiansen J et al. Multi detector computed tomography (MDCT) of the aortic root; ECG-gated verses non-ECG-gated examinations. Radiography $2011 ; 17: 218-222$ 